\title{
Levantamento da diversidade da macrofauna do solo no bosque das arapiracas
}

\section{Survey of the diversity of soil macrofauna in the arapiracas forest}

\author{
Carlos Petrúcio Silva dos Santos ${ }^{(1)}$; Rubens Pessoa de Barros ${ }^{(2)}$; Dayani Mayara Melo Nunes \\ Silva $^{(3)}$; Sâmilla Keisy dos Santos Moura ${ }^{(4)}$; Daniel Rocha Santos ${ }^{(5)}$ \\ (1)Graduando em Ciências Biológicas pela Universidade Estadual de Alagoas - UNEAL; Arapiraca, Alagoas; E- \\ mail:carlos.santos4@alunos.uneal.edu.br \\ (2)Professor do Departamento de Ciências Biológicas da Universidade Estadual de Alagoas - UNEAL; \\ Arapiraca, Alagoas;Email:Pessoa.rubens@gmail.com \\ (3)Graduando em Ciências Biológicas pela Universidade Estadual de Alagoas - UNEAL; Arapiraca, Alagoas; \\ Email:dayanimayara2@gmail.com \\ (4)Graduando em Ciências Biológicas pela Universidade Estadual de Alagoas - UNEAL. Arapiraca, Alagoas; \\ Email: samillamoura@alunos.uneal.edu.br \\ (5)Graduando em Ciências Biológicas pela Universidade Estadual de Alagoas - UNEAL; Arapiraca, Alagoas; \\ Email:danielrocha-100@outlook.com
}

Resumo - O solo é constituído pela associação de diversos elementos, como água, nutrientes e matéria orgânica que auxiliam no desenvolvimento dos seres vivos. A fauna edáfica do solo é responsável pela manutenção e sobrevivência do ambiente agindo nos processos de decomposição da matéria orgânica e ciclagem dos nutrientes, contribuindo para a fertilidade, equilíbrio e como um excelente indicador de qualidade. Sendo assim, o objetivo deste trabalho foi realizar uma análise quantitativa e identificar a distribuição dos macrooganismos do solo nos Bosque das Arapiracas. Foram coletas 20 amostras de serapilheira através da técnica de transecto, compreendendo assim, uma parcela amostral das áreas de um ecossistema. O material coletado foi triado manualmente, separando as espécies da macrofauna do substrato, e posteriormente, armazenando-as em frascos com álcool $70 \%$ e identificado ao nível de ordem taxonômica com auxílio de literaturas.

Palavras-chave: fauna edáfica. Invertebrados. Bioindicadores.

\begin{abstract}
The Soil is constituted by the association of several elements, such as water, nutrients and organic matter that help in the development of living beings. The soil edaphic fauna is responsible for the maintenance and survival of the environment, acting in the processes of organic matter decomposition and nutrient cycling, contributing to fertility, balance and as an excellent indicator of quality. Thus, the objective of this work was to perform a quantitative analysis and identify the distribution of soil macroorganisms in Bosque das Arapiracas. Twenty samples of litter were collected using the transect technique, thus comprising a sample of the areas of an ecosystem. The collected material was manually sorted, separating the macrofauna
\end{abstract}




\section{Revista Ambientale \\ Revista da Universidade Estadual de Alagoas/UNEAL \\ e-ISSN 2318-454X, Ano 13, Vol. 13 (2), 2021}

species from the substrate, and later, storing them in bottles with $70 \%$ alcohol and identified at the taxonomic level with the help of literature.

Keywords: edaphic fauna. Invertebrates. bioindicators.

\section{Introdução}

O solo é um ecossistema complexo constituído pela associação de diversos elementos que auxiliam na organização de suas partículas e na decomposição de resíduos orgânicos, assim como também, um grande responsável por fornecer substâncias que propiciam o desenvolvimento das plantas e animais que dependem direta e indiretamente dele, como água, nutrientes e a matéria orgânica (SILVA et al., 2016).

Muitos processos biológicos importantes para a manutenção da vida na Terra ocorrem no solo, sendo assim, uma ferramenta que desempenha uma grande variedade de funções vitais, de caráter ambiental, ecológico, social e econômico. A comunidade de organismos micro e macroscópicos que habitam o solo contribuem para essa manutenção e sobrevivência do ambiente. Entre esses processos pode-se destacar a formação de agregados, decomposição da matéria orgânica, a produção de húmus, a ciclagem de nutrientes e energia, o controle biológico de pragas e de doenças, produção de metabólitos secundários (antibióticos, hormônios, ácidos orgânicos, substâncias alelopáticas, e outros), a decomposição de resíduos tóxicos, a purificação da água e a produção de alimentos (BIGNELL et al., 2010; KORASAKI et al., 2013).

De acordo com Swift et al., 2010, a fauna do solo compreende os invertebrados que vivem no solo durante toda a vida ou em algum estágio do ciclo biológico, sendo esses, organismos classificados conforme o seu tamanho corporal. Assim, essa comunidade de indivíduos é dividida em Microfauna (protozoários e nematoides), Mesofauna (ácaros e colêmbolos) e a Macrofauna (moluscos, anelídeos e artrópodes).

A fauna do solo, particularmente, a macrofauna possui uma funcionalidade edáfica que é utilizada na avaliação do solo através da diversidade da biota, sendo relacionada até mesmo com a qualidade, física e biológica (TOLEDO, 2018). Esse grupo é constituído pelos invertebrados com mais de $10 \mathrm{~mm}$ de comprimento e/ou $2 \mathrm{~mm}$ de diâmetro, e são componentes importantes da biota do solo, pois exercem um papel fundamental na fragmentação do material vegetal e na regulação indireta dos processos biológicos do solo (AQUINO et al., 2008; BROWN et al., 2009; MELO et al., 2009; BARETTA et al., 2011).

Segundo Huber \& Morselli (2011), os organismos da macrofauna edáfica também são bastante consideráveis para o sistema de produção agrícola, onde contribuem diretamente para sua avaliação. A partir das informações obtidas por meio desses indivíduos, seu conhecimento pode ser de grande colaboração no desenvolvimento de estratégias de recuperação do solo ou mitigação de danos causados ao mesmo (ROVEDDER et al., 2009). Dessa forma, o levantamento e identificação desses indivíduos nas áreas, podem ser usados como bioindicadores do uso do solo e de sua fertilidade (REZENDE et al., 2017). 
A fauna do solo tem papel importante na avaliação das atividades promovidas pelo homem, sendo uma propriedade indicativa para monitorar a qualidade do ambiente (BARETTA et al., 2011). Sendo assim, o conhecimento e identificação dos organismos da macrofauna edáfica de uma área é de grande importância, pois, possibilitam uma maior exploração ambiental e auxiliam no funcionamento do equilíbrio do solo. Partindo disto, o trabalho teve como objetivo realizar o levantamento e avaliar a diversidade de macrofauna do solo no bosque das Arapiracas no município de Arapiraca - AL.

\section{Material e métodos}

O presente trabalho foi realizado no Bosque das Arapiracas (784Q+WW Capiata, Arapiraca - AL), localizado no Capiatã, no município de Arapiraca, Alagoas. O espaço possui vários plantios de árvores em seu interior, assim como uma grande extensão de pista de cooper e ciclovia que atrai muitos usuários para a prática de atividade física e lazer. De acordo com Gomes Júnior (2016, p. 55) Essa obra teve como objetivo "[...] recuperar e preservar as nascentes do rio Riacho Seco, ao mesmo tempo dotando a cidade de um pulmão verde e recompondo a vegetação das margens da antiga Lagoa das Olarias [...]”. A localização do ambiente a ser analisado pode ser vista na figura 4.

Figura 1. Localização do espaço estudado.

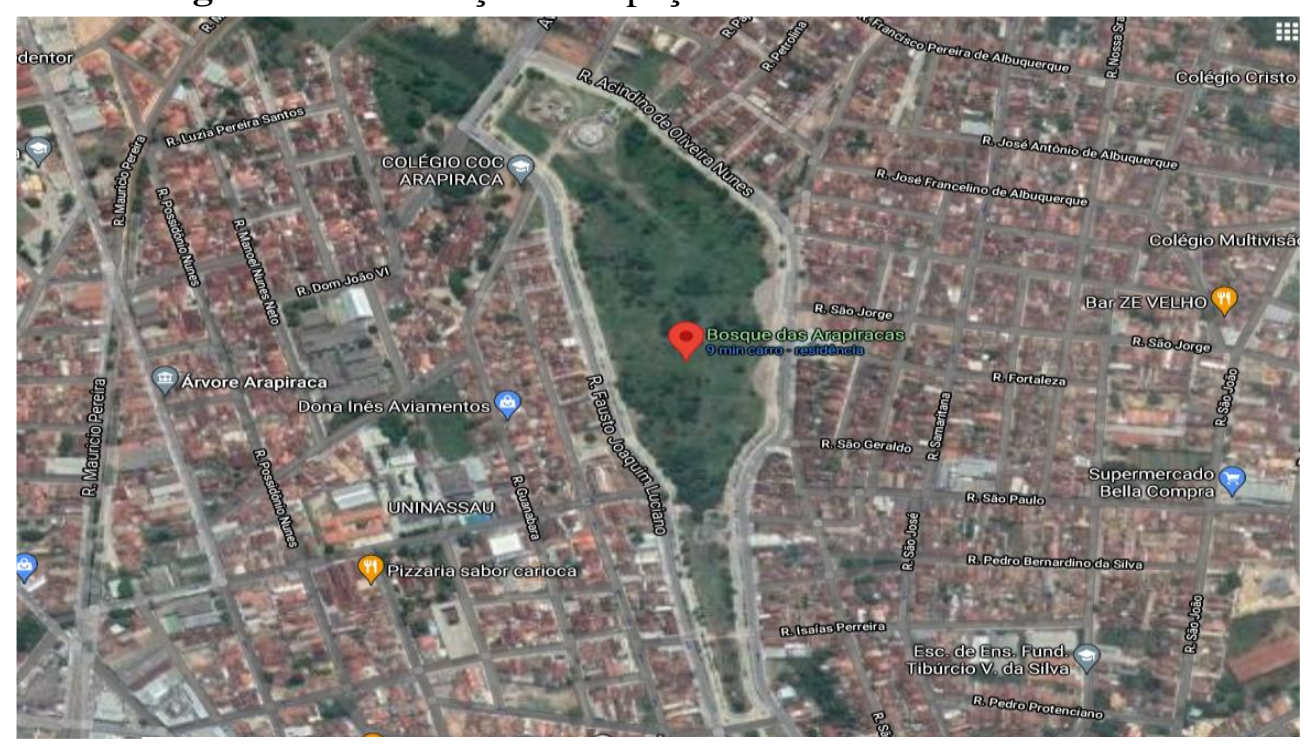

Fonte: https://www.google.com.br/maps, acessado em 2021.

$\mathrm{O}$ método utilizado foi o de transectos com de $50 \mathrm{~cm}^{2}$. Esse método consiste em coletar a serrapilheira que se encontra na superfície do local de estudo. foram coletadas 20 amostras de serrapilheira em dois dias de coleta, 10 em cada dia. Com as coletas podemos avaliar a macrofauna edáfica que podemos encontrar na região. Após a coleta, fizemos a triagem dos materiais encontrados em cada sacola de coleta, onde desenvolvida de forma manual a serrapilheira foi colocado em uma mesa na cor branca com uma luz branca para facilitar a identificação das espécies. 
As espécies encontradas foram separadas e fixadas em álcool $70 \%$ para a conservação, quantificação e identificação em nível de ordem. Deste modo, a técnica foi aplicada ás características ecológicas presentes na distribuição espacial, demonstrando, sobretudo, a riqueza da biota nesta parcela do bosque de Arapiraca-AL.

\section{Resultados e discussão}

Com isto, o trabalho seguiu as seguintes etapas aplicadas para a coleta de todo matéria biológica do local: 1) retirada da serapilheira em uma área de $50 \mathrm{~cm}^{2}$; 2) extração manual de todo material biológico; 3) triagem da amostra e separação da macrofauna e 4) contagem e identificação dos animais, como observa-se conforme as tabelas 1 e 2.

Tabela 1- Primeira coleta em 14/04/21 da macrofauna no bosque de Arapiraca-AL, dividida em 10 vezes.

\begin{tabular}{|c|c|c|c|c|c|c|c|c|c|c|c|}
\hline $\begin{array}{c}\text { PESO } \\
\text { (Kg) }\end{array}$ & $\mathbf{1}^{\mathbf{o}}$ & $\mathbf{2}^{\mathbf{o}}$ & $\mathbf{3}^{\mathbf{o}}$ & $\mathbf{4}^{\mathbf{o}}$ & $\mathbf{5}^{\mathbf{o}}$ & $\mathbf{6}^{\mathbf{o}}$ & $\mathbf{7}^{\mathbf{o}}$ & $\mathbf{8}^{\mathbf{o}}$ & $\mathbf{9}^{\mathbf{o}}$ & $\mathbf{1 0}^{\mathbf{o}}$ & $\begin{array}{c}\text { Quantidade } \\
\text { Total }\end{array}$ \\
\hline Peso total & 4,17 & 3,54 & 2,67 & 1,13 & 1,03 & 2,37 & 2,01 & 1,64 & 2,25 & 2,17 & 22,94 \\
\hline Peso verde & & 0,70 & & & & 0,13 & & & & & 0,83 \\
\hline $\begin{array}{c}\text { Peso da } \\
\text { terra }\end{array}$ & 3,37 & 1,33 & 1,99 & 0,60 & 0,85 & 1,86 & 1,75 & 1,14 & 1,98 & 1,33 & 16,21 \\
\hline Peso Seco & 0,70 & 1,43 & 0,50 & 0,48 & 0,14 & 0,33 & 0,26 & 0,25 & 0,30 & 0,77 & 5,18 \\
\hline $\begin{array}{c}\text { Quantidade } \\
\text { de Insetos }\end{array}$ & 1 & & & 1 & & & & 1 & 3 & 1 & 7 \\
\hline
\end{tabular}

Fonte: Elaborada pelos autores, 2021.

Tabela 2- Segunda coleta em 28/04/21 da macrofauna no bosque de Arapiraca-AL, dividida em 10 vezes.

\begin{tabular}{|c|c|c|c|c|c|c|c|c|c|c|c|}
\hline PESO (Kg) & $\mathbf{1}^{\mathbf{o}}$ & $\mathbf{2}^{\mathbf{o}}$ & $\mathbf{3}^{\mathbf{o}}$ & $\mathbf{4}^{\mathbf{o}}$ & $\mathbf{5}^{\mathbf{o}}$ & $\mathbf{6}^{\mathbf{o}}$ & $\mathbf{7}^{\mathbf{o}}$ & $\mathbf{8}^{\mathbf{o}}$ & $\mathbf{9}^{\mathbf{o}}$ & $\mathbf{1 0}^{\mathbf{o}}$ & $\begin{array}{c}\text { Quantida } \\
\text { de Total }\end{array}$ \\
\hline Peso total & 2,27 & 0,54 & 1,28 & 1,10 & 0,30 & 1,14 & 0,31 & 1,56 & 1,18 & 0,76 & 10,71 \\
\hline Peso verde & & & 0,02 & 0,08 & & & & 0,10 & 0,11 & & 0,32 \\
\hline $\begin{array}{c}\text { Peso da } \\
\text { terra }\end{array}$ & 0,81 & 0,44 & 0,90 & 0,94 & 0,21 & 0,97 & 0,20 & 1,35 & 0,48 & 0,62 & 6,95 \\
\hline Peso seco & 1,33 & 0,09 & 0,45 & 0,05 & 0,09 & 0,16 & 0,10 & 0,21 & 0,57 & 0,17 & 3,23 \\
\hline $\begin{array}{c}\text { Quantidade } \\
\text { de insetos }\end{array}$ & 31 & 0 & 0 & 0 & 51 & 61 & 0 & 56 & 15 & 10 & 224 \\
\hline
\end{tabular}

Fonte: Elaborada pelos autores, 2021. 
Foram encontrados 231 indivíduos na amostra de serapilheira da área estudada, pertencentes a sete Ordens (Tabela 3).

Tabela 3. Identificação da população de invertebrados no solo do bosque de Arapiraca-AL, classificados ao nível de família e Ordem.

\begin{tabular}{|c|c|c|c|c|}
\hline Nome popular & Nome científico & Família & Ordem & Quantidade \\
\hline Barata- silvestre & Ectobiussylvestris & Blattellidae & Blattodea & 6 \\
\hline Barata do suriname & Pycnoscelussurinamensis & Blaberidae & Blattodea & 2 \\
\hline $\begin{array}{l}\text { Besouro de despensa } \\
\text { (larva) }\end{array}$ & Dermestesmaculatus & Dermestidae & Coleoptera & 15 \\
\hline $\begin{array}{l}\text { Borboleta-caveira } \\
\text { (pupa) }\end{array}$ & Acherontiaatropos & Sphingidae & Lepidoptera & 1 \\
\hline Broca-das-peles & Attagenuspellio & Dermestidae & Coleoptera & 40 \\
\hline Cigarra & Carinetafasciculata & Cicadidae & Hemiptera & 3 \\
\hline $\begin{array}{l}\text { Embuá/ piolho de } \\
\text { cobra }\end{array}$ & Lulus sabulosuscylindroiulus & Julidae & Juliformia & 5 \\
\hline Larva- arame & Conoderusscalaris & Elateridae & Coleoptera & 1 \\
\hline Mosca soldado negra & Hermetiaillucens & Stratiomyidae & Diptera & 5 \\
\hline Tatuzinho de Jardim & ArmadillidiumVulgare & & Isopoda & 153 \\
\hline
\end{tabular}

Fonte: Elaborada pelos autores, 2021.

Utilizando o método de triagem manual, a ordem Isopoda apresentou maior ocorrência no ambiente analisado, particularmente da família Armadillidiidae. A espécie encontrada através da técnica do transecto, foi a de nome popularmente conhecida como tatuzinhos-dejardim (Armadillidiumvulgare), estes recebem tal nominação devido à capacidade de se curvarem em situações de perigo, formando uma estrutura esférica (CORREIA, 2008). Nos ecossistemas onde ocorrem os isópodes, há uma ativa participação na ciclagem dos nutrientes que são absorvidos pelas plantas, devido a sua capacidade de serem detritívoros eficientes e macro decompositores, ou seja, geralmente se alimentam de plantas mortas e em decomposição, fragmentando a serapilheira e acelerando o processo de decomposição (ARAUJO, 1999; QUADROS, 2009). Assim conseguimos ver a importância dessa espécie para o ecossistema, pois o local de estudo possui grande quantidade de material orgânico em decomposição.

A segunda ordem encontrada em maior densidade neste estudo foi a Coleóptera, representada por besouros, esses indivíduos são benéficos como bioindicadores por serem altamente influenciados pelo meio, que afeta diretamente a distribuição destes indivíduos devido à variedade de seus hábitos alimentares, nichos ecológicos, riqueza de famílias e diversidade de espécies (TEIXEIRA; HOFFMANN; SILVA-FILHO, 2009). 
Desse modo, a coleta do solo do bosque das Arapiracas mostrou-se significativamente positiva para amostra de matérias biológicas que possibilitam o estudo da biodiversidade de macro-organismos. Sugere-se também através desse estudo, a utilização desses invertebrados como bioindicadores, podendo ser utilizados para a avaliação da qualidade do ambiente, principalmente em áreas de recuperação ambiental, o que pode ser o caso de um sistema florestal.

\section{Conclusão}

A coleta resultou em 231 invertebrados pertencentes a sete ordens, com maior abundância na ordem Isopoda. Sendo assim, o conhecimento desses organismos presentes no município de Arapiraca, pode auxiliar e esclarecer seu papel potencial nas propriedades de solo, contribuindo para futuros estudos em torno da sua importância e atuação como agentes bioindicadores.

\section{Conflitos de interesse}

Os autores deste manuscrito não declararam conflitos de interesse.

\section{Referências}

AQUINO, A. M. de; CORREIA, M. E. F.; ALVES, M. V. Diversidade da macrofauna edáfica no Brasil. In: MOREIRA, F. M. S.; SIQUEIRA, J. O.; BRUSSAARD, L. (Eds.). Biodiversidade do solo em ecossistemas tropicais. Lavras: Editora da UFLA, p. 143-170. 2008.

ARAUJO, Paula Beatriz. Subordem Oniscidea (isopodos terrestres, "tatuzinhos"). In L. BUCKUP \& G. BOND-BUCKUP Eds. Os Crustáceos do Rio Grande do Sul. Porto Alegre, Editora: Universidade /UFRGS, 503p. 1999.

BARETTA, D.; SANTOS, J. P. C.; SEGAT, J. C.; GEREMIA, E. V.; OLIVEIRAFILHO, L. C. L.; ALVES, M. V. Fauna edáfica e qualidade do solo. In: KLAUBERGFILHO, O.; MAFRA, A. L.; GATIBONI, L. C. Tópicos em Ciências do solo. Viçosa: Sociedade Brasileira de Ciência do Solo, v. 7, p. 141-192, 2011. 
BIGNELL, D.; CONSTANTINO, R.; CSUDI, C.; KARYANTO, A.; KONATÉ, S.; LOUZADA, J. N. C.; SUSILO, F. X.; TONDOH, J. E.; ZANETTI, R. Macrofauna. In: MOREIRA, F. M. S.; HUISING, E. J.; BIGNELL, D. E. (Eds.). Manual de biologia dos solos tropicais: amostragem e caracterização da biodiversidade. Lavras: Editora da UFLA, p.121-137.2010.

BROWN, G. G.; MASCHIO, W.; FROUFE, L. C. M. Macrofauna do solo em sistemas agroflorestais e Mata Atlântica em regeneração nos municípios de Barra do Turvo, SP, e Adrianópolis, PR. Colombo: Embrapa Florestas, 2009. 51 p.

CORREIA, Maria Elizabeth Fernandes; AQUINO, Adriana Maria de; AGUIAR-MENEZES, Elen de Lima. Aspectos Ecológicos dos Isopoda Terrestres. Embrapa- Agrobiologia, Documentos, 249, 2008.

GOMES JÚNIOR, José de Souza. Produção e uso do espaço público em Arapiraca, Alagoas: uma avaliação pós-ocupação do parque Municipal Ceci Cunha e do Bosque das Arapiracas.2016.119f. Dissertação (Mestrado em Arquitetura e Urbanismo: Dinâmicas do Espaço Habitado)-Faculdade de Arquitetura e Urbanismo,Universidade Federal de Alagoas, Maceió, 2016.

HUBER, A. C. K.; MORSELLI, T. B. G. A. Estudo da mesofauna (ácaros e colêmbolos) no processo da vermicompostagem. Revista da FZVA. Uruguaiana, v. 18, n. 2, p. 12-20, 2011.

KORASAKI, V.; MORAIS, J. W. de; BRAGA, R. F. Macrofauna. In: MOREIRA. F. M. S.; CARES, J. E.; ZANETTI, R.; STÜRMER, S. L. (Eds.). O ecossistema solo: componentes, relações ecológicas e efeitos na produção vegetal. Lavras: Editora da UFLA, p. 79-128.2013.

MELO, F. V.; BROWN, G. G.; CONSTANTINO, R.; LOUZADA, J. N. C.; LUIZÃO, F. J.; MORAIS, J. W.; ZANETTI, R. A. importância da meso e macrofauna do solo na fertilidade e como biondicadores. Boletim Informativo da SBCS, p. 38-43, jan/abr., 2009.

QUADROS, A. F. Ecologia populacional, estratégias reprodutivas e uso de recursos por isópodos terrestres neotropicais (Crustacea, Isopoda). Tese (Doutorado em Biologia animal) -Instituto de Biociências.Universidade Federal do Rio Grande do Sul. 2009.

REZENDE, Leandro Pereira; PORTELA, Grazielly de França; MACEDO, Natália Cordeiro; DINIZ, Kerollayne Dourado. Identificação da Macrofauna do Solo em pastagem de PanicumMaximum JACQ. e área submetida à queimada no Município de Sambaíba- MA. Revista Biodiversidade, V. 16, n. 1, p.21- 32, 2017.

ROVEDDER, A. P. M.; ELTZ, F. L. F.; DRESCHER, M. S.; SCHENATO, R. B.; ANTONIOLLI, Z. I. Organismos edáficos como bioindicadores da recuperação de solos degradados por arenização no Bioma Pampa. Ciência Rural, v. 39, n. 4, p. 1061-1068, 2009. 
SILVA, F. C.; SANTANA, Í. J.; MARTINS, R. D.; LEMES, N. M.; RIETJENS, A. R.; LIMA, M. L. P. Quantificação da microbiota e diversidade ecológica da meso e macrofauna do solo sob diferentes usos no município de Urutaí (região Sudeste Goiano). Multi-ScienceJournal, Goiânia, v.1, n.4, p.12-18. 2016.

SWIFT, M. J.; BIGNELL, D.; MOREIRA, F. M. de S.; HUISING, J. O inventário da biodiversidade biológica do solo: conceitos e orientações gerais. In: MOREIRA, F. M. S.; HUISING, E. J.; BIGNELL, D. E. (Eds.). Manual de biologia dos solos tropicais: amostragem e caracterização da biodiversidade. Lavras: Editora da UFLA, p. 23-41. 2010.

TEIXEIRA, C. C. 1.; HOFFMANN, M.; SILVA-FILHO, G. Comunidade de Coleoptera de solo em remanescente de Mata Atlântica no estado do Rio de Janeiro, Brasil. Biota Neotrópica, São Paulo, v. 9, n. 4, p. 91-95, 19 nov. 2009.

TOLEDO, Vinicius Guimarães; SANTOS, Bruna de Assumpção dos; SANTOS, Everaldo dos. Levantamento da Macrofauna Invertebrada do Solo Sob Três Diferentes Sistemas de Cobertura Vegetal na Floresta Ombrófila Densa das Terras Baixas no Litoral do Paraná. IX Congresso Brasileiro de Gestão Ambiental, 2018. 